Article

\title{
Best Proximity Points for Alternative Maps
}

\author{
Yi Chou Chen (1)
}

Department of General Education, National Army Academy, Taoyuan City 32093, Taiwan; cycu.chou@gmail.com

Received: 13 April 2019; Accepted: 30 May 2019; Published: 3 June 2019

check for updates

\begin{abstract}
Let $(\mathfrak{X}, \mathfrak{d})$ be a metric space and $\Omega_{i}, i=1,2, \ldots, m$, be a nonempty subset of $(\mathfrak{X}, \mathfrak{d})$. An operator $T: \cup_{1 \leq i \leq m} \Omega_{i} \rightarrow \cup_{1 \leq i \leq m} \Omega_{i}$ is called an alternative map if $T\left(\Omega_{j}\right) \subseteq \cup_{i \neq j} \Omega_{i}$, $j=1,2, \ldots, m$. In addition, if for any $x, y \in \cup_{1 \leq i \leq m} \Omega_{i}$, there exists a constant $\alpha \in[0,1)$ such that $\mathfrak{d}(T x, T y) \leq \alpha \mathfrak{d}(x, y)+(1-\alpha) \mathfrak{d}\left(\Omega_{j}, \Omega_{k}\right)$ for some $\Omega_{j}$ and $\Omega_{k} \in\left\{\Omega_{i}\right\}_{i=1}^{m}$ with $x \in \Omega_{j}$ and $y \in \Omega_{k}$, then we call $T$ an alternative contraction. Moreover, if $(\mathfrak{X}, \mathfrak{d})$ has an alternative UC property and $T$ is an alternative contraction, then the best proximity point of $T$ exists.
\end{abstract}

Keywords: alternative map; best proximity point; convergence theorem

\section{Introduction}

When viewing the topic of $n$-sets methodology, it can be seen that many authors will often create contractions that consider only a sequential pattern in their published findings.

However, Kirk et al. (2003) [1] , and Eldred and Veeramani [2] (2006)'s 2-sets methodologies which both use a sequential pattern also allows for this paper's newly proposed "alternative n-sets" contraction to be defined as a special case in which we may now consider that the map of point does not need to follow a sequential pattern. Additionally, this alternative n-sets contraction in conjunction with Suzuki et al. [3] (2009)'s UC Property and Fan [4] (1969)'s Best Proximity Points allows for the defining of a "New UC Condition" and non-sequential best proximity points.

It is this paper's purpose to explore this idea of a non-sequential n-sets methodology and inspire new possibilities with said idea.

Definition 1 (Kirk et al. [1]). Let $(\mathfrak{X}, \mathfrak{d})$ be a metric space, $\Omega_{i}$ be nonempty subsets of $\mathfrak{X}, i=1,2, \ldots, m$. A map $T: \cup_{i=1}^{m} \Omega_{i} \rightarrow \cup_{i=1}^{m} \Omega_{i}$ is a cyclic map if $T\left(\Omega_{i}\right) \subseteq \Omega_{i+1}$ for $i=1,2, \ldots, m$ and $\Omega_{m+1}=\Omega_{1}$.

The map proposed by Kirk et al. may follow a certain fixed path; that is, the point always is sent by an operator $T$ from one set to another sequentially. Moreover, Eldred and Veeramani gives a contraction on the two subsets of $\mathfrak{X}$ in 2006 as follows:

Definition 2 (Eldred and Veeramani [2]). Let $(\mathfrak{X}, \mathfrak{d})$ be a metric space, and let $\mathfrak{A}$ and $\mathfrak{B}$ be nonempty subsets of $\mathfrak{X}$. A map $T: \mathfrak{A} \cup \mathfrak{B} \rightarrow \mathfrak{A} \cup \mathfrak{B}$ is a cyclic map if $T(\mathfrak{A}) \subseteq \mathfrak{B}$ and $T(\mathfrak{B}) \subseteq \mathfrak{A}$. A map $T: \mathfrak{A} \cup \mathfrak{B} \rightarrow \mathfrak{A} \cup \mathfrak{B}$ is a cyclic contraction if there exists $\alpha \in[0,1)$ satisfying

$$
\mathfrak{d}(T x, T y) \leq \alpha \mathfrak{d}(x, y)+(1-\alpha) \mathfrak{d}(\mathfrak{A}, \mathfrak{B}),
$$

for all $x \in \mathfrak{A}, y \in \mathfrak{B}$.

The contribution of Eldred and Veeramani was to create the cyclic contraction. Many authors have created several different contractions by modifying Eldred and Veeramani; see e.g., [5-18]. In this 
paper, we define a new map which is concerned with the alternative $n$-sets methodology instead of 2-sets or sequentially $n$-sets as follows:

Definition 3. Let $(\mathfrak{X}, \mathfrak{d})$ be a metric space, $\Omega_{i}, i=1,2, \ldots, m$ be nonempty subsets of $(\mathfrak{X}, \mathfrak{d})$. A map $T: \cup_{i=1}^{m} \Omega_{i} \rightarrow \cup_{i=1}^{m} \Omega_{i}$ is called an alternative map if $T\left(\Omega_{i}\right) \subseteq \cup_{j \neq i} \Omega_{i}$, for $i=1,2, \ldots, m$. Next, $T$ is defined as an alternative contraction (AC) if $T$ is an alternative map and there exists a constant $\alpha \in[0,1)$ such that for any $x \in \Omega_{j}, y \in \Omega_{k}$ for some $\Omega_{j}, \Omega_{k} \in\left\{\Omega_{i}\right\}_{i=1}^{m}$, the following condition holds:

$$
\mathfrak{d}(T x, T y) \leq \alpha \mathfrak{d}(x, y)+(1-\alpha) \mathfrak{d}\left(\Omega_{j}, \Omega_{k}\right) .
$$

Now, consider Suzuki et al. [3]'s UC condition:

Definition 4 (Suzuki et al. [3]). Let $A$ and $B$ be nonempty subsets of a metric space $(\mathfrak{X}, \mathfrak{d})$. Then $(A, B)$ is said to satisfy the property UC if the following holds: If $\left\{x_{n}\right\}$ and $\left\{x_{n}^{\prime}\right\}$ are sequences in $A$ and $\left\{y_{n}\right\}$ is a sequence in $B$ such that $\lim _{n} \mathfrak{d}\left(x_{n}, y_{n}\right)=\mathfrak{d}(\mathfrak{A}, \mathfrak{B})$ and $\lim _{n} \mathfrak{d}\left(x_{n}^{\prime}, y_{n}\right)=\mathfrak{d}(\mathfrak{A}, \mathfrak{B})$, then $\lim _{n} \mathfrak{d}\left(x_{n}, x_{n}^{\prime}\right)=0$ holds.

The UC condition only takes into consideration Eldred and Veeramani [2]'s 2-sets model. Taking into account the above alternative contraction, an alternative UC Condition may be created:

Definition 5 (Alternative UC condition). Let $(\mathfrak{X}, \mathfrak{d})$ be a metric space and $\Omega_{i}, i=1,2, \ldots, m$ be nonempty subsets of $\mathfrak{X}$. If $\left\{\Omega_{i}\right\}_{i=1}^{m}$ is said to satisfy the UC condition if the following holds. Let $y_{n}^{1}, y_{n}^{v} \in A_{r}$ and $y_{n}^{2}, y_{n}^{3}, \ldots, y_{n}^{v-1} \notin \cup_{j \neq r} \Omega_{j}$ for some positive integer $v \geq 2$. If $\lim _{n \rightarrow \infty} \mathfrak{d}\left(y_{n}^{1}, y_{n}^{2}\right)=\lim _{n \rightarrow \infty} \mathfrak{d}\left(y_{n}^{2}, y_{n}^{3}\right) \ldots=$ $\lim _{n \rightarrow \infty} \mathfrak{d}\left(y_{n}^{v-1}, y_{n}^{v}\right)=\mathfrak{d}(\mathfrak{A}, \mathfrak{B})$ for some $\mathfrak{A}, \mathfrak{B} \in\left\{\Omega_{i}\right\}_{i=1}^{m}$, then $\lim _{n \rightarrow \infty} \mathfrak{d}\left(y_{n}^{1}, y_{n}^{v}\right)=0$.

One can easily see that a cyclic map is a special case of an alternative map.

Due to Fan [4], many authors publish their papers with their Best Proximity Points in [19-34]:

Definition 6. Let $(\mathfrak{X}, \mathfrak{d})$ be a metric space and $\mathfrak{A}, \mathfrak{B}$ be nonempty subsets of $\mathfrak{X}$. If $p \in \mathfrak{X}$ is called a best proximity point of a cyclic map $T$ if

$$
\mathfrak{d}(T p, p)=\mathfrak{d}(\mathfrak{A}, \mathfrak{B}) .
$$

Using the alternative UC condition and the alternative contraction, we may define new best proximity points:

Definition 7. Let $(\mathfrak{X}, \mathfrak{d})$ be a metric space and $\Omega_{i}, i=1,2, \ldots, m$, be nonempty subsets of $\mathfrak{X}$. If $p \in X$ is called a best proximity point of an alternative map $T$ if there exists a positive integer $\ell(\geq 2), \Omega_{r_{j}} \in\left\{\Omega_{i}\right\}_{i=1}^{m}$ for some $j=0,1,2, \ldots, \ell-1$ and $p \in \Omega_{r_{0}}, T p \in \Omega_{r_{1}}, T^{2} p \in \Omega_{r_{2}}, \ldots$, and $T^{\ell-1} p \in \Omega_{r_{\ell-1}}$ satisfying the following three conditions:

1. $p=T^{\ell} p$,

2. $\mathfrak{d}\left(T^{i} p, T^{i+1} p\right)=\mathfrak{d}\left(\Omega_{r_{i}}, \Omega_{r_{i+1}}\right), i=0,1,2, \ldots, \ell-1\left(\right.$ Here $\left.T^{0} p=p\right)$,

3. $\quad \mathfrak{d}\left(\Omega_{r_{0}}, \Omega_{r_{1}}\right)=\mathfrak{d}\left(\Omega_{r_{1}}, \Omega_{r_{2}}\right)=\cdots=\mathfrak{d}\left(\Omega_{r_{\ell-1}}, \Omega_{r_{0}}\right)$.

The traditional cyclic map can be used to deal with the best proximity points of a model using two mirrors. Based on Eldred and Veeramani [2]'s cyclic map, the cyclic contraction is formulated to specify the path that the light will follow from one mirror to next. Suzuki et al. [32] proves that under cyclic contraction and the UC condition, there will be certain points reflected many times between the two mirrors; these points, called the best proximity points, are bounced infinite times, creating particularly bright points. This paper's alternative map changes the model from a two-mirror map into one concerning numerous mirrors. In this model, after the light is reflected from a mirror, the light will not necessarily return to said mirror and its path may diverge to any given point. According to the 
variance of the angle of refraction, it is impossible to know to which mirror the light will be reflected to next. Nevertheless, in this article we prove when light is reflected many times under the conditions of the proposed alternative contraction and the UC condition, in some mirrors, these points will be become particularly shining points, or the best proximity points of the alternative map.

Theorem 5 proves that if $T$ is an alternative contraction, some proximity points exist. Some preparations presented in Sections 2 and 3 explain the reasoning behind this proof. Finally, we may assert that the main results are not unusual since it can be applied to both compact space and complete metric space.

\section{Alternative Contractions}

We rewrite the alternative maps in Definition 3 as if for any $x \in \Omega_{i}$, then $T x \in \Omega_{j}$, for some $j \neq i$. Next, we introduce two weaker conditions of the alternative contraction (AC): alternative decreasing contraction (ADC) and alternative limiting contraction (ALC) as below.

Definition 8. Let $(\mathfrak{X}, \mathfrak{d})$ be a metric space and $\Omega_{i}, i=1,2, \ldots, m$, be nonempty subsets of $\mathfrak{X}$.

- $\quad T$ is called an alternative decreasing contraction (ADC) if for any $x \in \cup_{i=1}^{m} \Omega_{i}$, there exists a constant $\alpha \in[0,1)$ satisfying

$$
\mathfrak{d}\left(T^{2} x, T x\right) \leq \alpha \mathfrak{d}(T x, x)+(1-\alpha) \mathfrak{d}\left(\Omega_{j}, \Omega_{k}\right),
$$

with $T x \in \Omega_{j}, x \in \Omega_{k}$ for some different $j, k$.

- $T$ is called an alternative limiting contraction (ALC) if the following two conditions holds: $(i)$ if the limit $\lim _{i \rightarrow \infty} T^{n_{i}} x$ exists for some $x \in \cup_{i=1}^{m} \Omega_{i}$ and some subsequence $\left\{n_{i}\right\}_{i=1}^{\infty}$ of $\mathbb{N}$, then

$$
\lim _{i \rightarrow \infty} \mathfrak{d}\left(T\left(\lim _{j \rightarrow \infty} T^{n_{j}} x\right), T^{n_{i}+1} x\right)=0 \text { holds. }
$$

Lemma 1. $A C$ is $A D C$.

Lemma 2. $A C$ is $A L C$.

Proof. Assume that $T: \cup_{i=1}^{m} \Omega_{i} \longrightarrow \cup_{i=1}^{m} \Omega_{i}$ is a AC. Since $\lim _{i \rightarrow \infty} T^{n_{i}} x$ exists for some $x \in \cup_{i=1}^{m} \Omega_{i}$ and some subsequence $\left\{n_{i}\right\}_{i=1}^{\infty}$ of $\mathbb{N}$ by assumption, it follows that $\lim _{i \rightarrow \infty} T^{n_{i}} x \in \mathfrak{A}$ for some $\mathfrak{A} \in\left\{\Omega_{i}\right\}_{i=1}^{m}$. In addition, since $x \in \cup_{i=1}^{m} \Omega_{i}$, we have $\left\{T^{n_{i}} x\right\}_{i=1}^{\infty} \subseteq\left\{\Omega_{i}\right\}_{i=1}^{m}$. By the pigeonhole principle, we can choose a subsequence $\left\{n_{i_{j}}\right\}_{j=1}^{\infty} \subseteq\left\{n_{i}\right\}_{i=1}^{\infty}$ and $\Omega_{s}$ for some $s$ such that $T^{n^{i}}{ } x \in \Omega_{s}$ for all $j \in \mathbb{N}$. Since $\left\{n_{i_{j}}\right\}_{j=1}^{\infty}$ is a subsequence of $\left\{n_{i}\right\}_{i=1}^{\infty}$ and $\lim _{i \rightarrow \infty} T^{n_{i}} x$ exists, we have $\lim _{j \rightarrow \infty} T^{n_{i_{j}}} x$ exists and $\lim _{j \rightarrow \infty} T^{n_{i j}} x=$ $\lim _{i \rightarrow \infty} T^{n_{j}} x$. It follows that

$$
\mathfrak{d}\left(\mathfrak{A}, \Omega_{s}\right) \leq \mathfrak{d}\left(\lim _{j \rightarrow \infty} T^{n_{j}} x, T^{n_{i j}} x\right) .
$$

By definitions of alternative contractions, then

$$
\begin{aligned}
\lim _{i \rightarrow \infty} \mathfrak{d}\left(T\left(\lim _{i \rightarrow \infty} T^{n_{i}} x\right), T^{n_{i}+1} x\right) & =\lim _{j \rightarrow \infty} \mathfrak{d}\left(T\left(\lim _{j \rightarrow \infty} T^{n_{j}} x\right), T^{n_{j}+1} x\right) \\
(\text { by AC }) & =\lim _{j \rightarrow \infty}\left(\alpha \mathfrak{d}\left(\lim _{j \rightarrow \infty} T^{n_{j}} x, T^{n_{j}} x\right)+(1-\alpha) \mathfrak{d}\left(\mathfrak{A}, \Omega_{S}\right)\right) \\
\leq & \lim _{j \rightarrow \infty}\left(\alpha \mathfrak{d}\left(\lim _{j \rightarrow \infty} T^{n_{j}} x, T^{n_{j}} x\right)+(1-\alpha) \mathfrak{d}\left(\lim _{j \rightarrow \infty} T^{n_{j}} x, T^{n_{i j}} x\right)\right) \\
& =\lim _{j \rightarrow \infty} \mathfrak{d}\left(\lim _{j \rightarrow \infty} T^{n_{j}} x, T^{n_{j}} x\right) \\
& =0 .
\end{aligned}
$$


Since the $\mathfrak{d}\left(T\left(\lim _{j \rightarrow \infty} T^{n_{j}} x\right), T^{n_{j}+1} x\right)$ is always nonnegative, we have

$$
\lim _{j \rightarrow \infty} \mathfrak{d}\left(T\left(\lim _{j \rightarrow \infty} T^{n_{j}} x\right), T^{n_{j}+1} x\right)=0
$$

Next, we introduce some properties of ADC as follows:

Theorem 1. Let $(\mathfrak{X}, \mathfrak{d})$ be a metric space and $\Omega_{i}, i=1,2, \ldots, m$, nonempty subsets of $\mathfrak{X}$. If $T: \cup_{i=1}^{m} \Omega_{i} \longrightarrow$ $\cup_{i=1}^{m} \Omega_{i}$ is an alternative contraction, then for any $x \in \cup_{i=1}^{m} \Omega_{i}$,

$$
\lim _{n \rightarrow \infty} \mathfrak{d}\left(T^{n+1} x, T^{n} x\right) \text { exists. }
$$

Proof. Since $T$ is an alternative map, for any $x \in \cup_{k=1}^{m} \Omega_{k}$ and any $n \in \mathbb{N}$, then $T^{n-1} x \in \Omega_{i(n-1)}$ (where $\left.T^{0} x \triangleq x\right)$, it follows that $T^{n} x=T\left(T^{n-1} x\right) \in \Omega_{i(n)}$, it can be easily checked that $\Omega_{i(n-1)} \neq \Omega_{i(n)}$. Moreover, because $T$ is an alternative contraction, there exists a constant $\alpha \in[0,1)$ such that

$$
\begin{aligned}
\mathfrak{d}\left(T^{n+1} x, T^{n} x\right) & =\mathfrak{d}\left(T^{2}\left(T^{n-1} x\right), T\left(T^{n-1} x\right)\right) \\
& \leq \alpha d\left(T\left(T^{n-1} x\right), T^{n-1} x\right)+(1-\alpha) \mathfrak{d}\left(\Omega_{i(n-1)}, \Omega_{i(n)}\right) \\
& =\alpha \mathfrak{d}\left(T^{n} x, T^{n-1} x\right)+(1-\alpha) \mathfrak{d}\left(\Omega_{i(n-1)}, \Omega_{i(n)}\right) .
\end{aligned}
$$

Furthermore, since $T^{n} x \in \Omega_{i(n)}$ and $T^{n-1} x \in \Omega_{i(n-1)}$, we have $\mathfrak{d}\left(\Omega_{i(n)}, \Omega_{i(n-1)}\right) \leq \mathfrak{d}\left(T^{n} x, T^{n-1} x\right)$ and it then

$$
\begin{aligned}
\mathfrak{d}\left(T^{n+1} x, T^{n} x\right) & \leq \alpha \mathfrak{d}\left(T^{n} x, T^{n-1} x\right)+(1-\alpha) \mathfrak{d}\left(\Omega_{i(n)}, \Omega_{i(n-1)}\right) \\
& \leq \alpha \mathfrak{d}\left(T^{n} x, T^{n-1} x\right)+(1-\alpha) \mathfrak{d}\left(T^{n} x, T^{n-1} x\right) \\
& =\mathfrak{d}\left(T^{n} x, T^{n-1} x\right),
\end{aligned}
$$

for any $n \in \mathbb{N}$. That is, $\left\{\mathfrak{d}\left(T^{n+1} x, T^{n} x\right)\right\}_{n \in \mathbb{N}}$ is a decreasing sequence. Furthermore, since $\mathfrak{d}\left(T^{n+1} x, T^{n} x\right)$ is bounded below by " 0 ", $\lim _{n \rightarrow \infty} \mathfrak{d}\left(T^{n+1} x, T^{n} x\right)$ exists by the bounded monotone theorem.

Corollary 1. If $T: \cup_{i=1}^{m} \Omega_{i} \longrightarrow \cup_{i=1}^{m} \Omega_{i}$ is an $A D C$, then for any $x \in \cup_{i=1}^{m} \Omega_{i},\left\{\mathfrak{d}\left(T^{n+1} x, T^{n} x\right)\right\}_{n \in \mathbb{N}}$ is a decreasing sequence.

\section{Best Proximity Points}

In this section, in order to give the definition of best proximity points of alternative maps, we would remind the definition of a best proximal point of a cyclic map which is proposed by Fan and we rewrite to be applied in the metric space below.

Definition 9 (Fan [4]). Let $(\mathfrak{X}, \mathfrak{d})$ be a metric space and $\mathfrak{A}, \mathfrak{B}$ be nonempty subsets of $\mathfrak{X}$. If $p \in \mathfrak{X}$ is called a best proximity point of a cyclic map $T$ if

$$
\mathfrak{d}(T p, p)=\mathfrak{d}(\mathfrak{A}, \mathfrak{B}) .
$$

Next, we would like to extend the above definitions to the alternative map as follows:

Definition 10. Let $(\mathfrak{X}, \mathfrak{d})$ be a metric space and $\Omega_{i}, i=1,2, \ldots, m$, be nonempty subsets of $\mathfrak{X}$. If $p \in X$ is called a best proximity point of an alternative map $T$ if there exists a positive integer $\ell(\geq 2), \Omega_{r_{j}} \in\left\{\Omega_{i}\right\}_{i=1}^{m}$ 
for some $j=0,1,2, \ldots, \ell-1$ and $p \in \Omega_{r_{0}}, T p \in \Omega_{r_{1}}, T^{2} p \in \Omega_{r_{2}}, \ldots$, and $T^{\ell-1} p \in \Omega_{r_{\ell-1}}$ satisfying the following three conditions:

1. $p=T^{\ell} p$,

2. $\quad \mathfrak{d}(p, T p)=\mathfrak{d}\left(\Omega_{r_{0}}, \Omega_{r_{1}}\right)$

$\mathfrak{d}\left(T p, T^{2} p\right)=\mathfrak{d}\left(\Omega_{r_{1}}, \Omega_{r_{2}}\right)$

$\mathfrak{d}\left(T^{2} p, T^{3} p\right)=\mathfrak{d}\left(\Omega_{r_{2}}, \Omega_{r_{3}}\right)$

$$
\mathfrak{d}\left(T^{\ell-1} p, T^{\ell} p\right)=\mathfrak{d}\left(\Omega_{r_{\ell-1}}, \Omega_{r_{0}}\right),
$$

3. $\quad \mathfrak{d}\left(\Omega_{r_{0}}, \Omega_{r_{1}}\right)=\mathfrak{d}\left(\Omega_{r_{1}}, \Omega_{r_{2}}\right)=\cdots=\mathfrak{d}\left(\Omega_{r_{\ell-1}}, \Omega_{r_{0}}\right)$.

From the above definitions, we know that the number $\ell$ is not unique. We give an example as follows:

Example 1. Let $\Omega_{1}=\left\{(x, y) \in \mathbb{R}^{2} \mid x-y \geq 1, x+y \geq 1\right\}, \Omega_{2}=\left\{(x, y) \in \mathbb{R}^{2} \mid x-y \leq 1, x+y \geq 1\right\}$, $\Omega_{3}=\left\{(x, y) \in \mathbb{R}^{2} \mid x-y \leq-1, x+y \leq-1\right\}, \Omega_{4}=\left\{(x, y) \in \mathbb{R}^{2} \mid x+y \leq-1, x-y \geq-1\right\}$. Let the map $T$ as

$$
T\left(\begin{array}{l}
x \\
y
\end{array}\right)=\frac{1}{2}\left(\begin{array}{cc}
0 & 1 \\
-1 & 0
\end{array}\right)^{\tau}\left\{\left(\begin{array}{l}
x \\
y
\end{array}\right)+\left(\begin{array}{l}
1 \\
0
\end{array}\right):\left(\begin{array}{l}
x \\
y
\end{array}\right) \in \mathbb{R}^{2}\right\},
$$

where $\tau$ is a random variable in $\left(4 \mathbb{N}^{+}+1\right) \cup\left(4 \mathbb{N}^{+}+2\right) \cup\left(4 \mathbb{N}^{+}+3\right)$.

At second, we will complete our works step by step as follows.

Theorem 2. Let $(\mathfrak{X}, \mathfrak{d})$ be a metric space and $\Omega_{i}, i=1,2, \ldots, m$, be nonempty subsets of $\mathfrak{X}$. Let $T: \cup_{i=1}^{m} \Omega_{i} \longrightarrow \cup_{i=1}^{m} \Omega_{i}$ be an alternative contraction, then for any $x \in \cup_{i=1}^{m} \Omega_{i}$, we have

$$
\lim _{n \rightarrow \infty} \mathfrak{d}\left(T^{n+1} x, T^{n} x\right) \in\left\{\mathfrak{d}\left(\Omega_{i}, \Omega_{j}\right)\right\}_{1 \leq i \neq j \leq m} .
$$

Proof. Assume that $T^{n} x \in \Omega_{i(n)}$, for some $i(n) \in I$, this would imply that $\Omega_{i(n-1)} \neq \Omega_{i(n)}$ for any $n$ because $T$ is an alternative map. Moreover, since $T$ is an alternative contraction, there exists a constant $\alpha$ such that

$$
\begin{aligned}
\mathfrak{d}\left(T^{n+2} x, T^{n+1} x\right) & =d\left(T\left(T^{n+1} x\right), T\left(T^{n} x\right)\right) \\
& \leq \alpha \mathfrak{d}\left(T^{n+1} x, T^{n} x\right)+(1-\alpha) \mathfrak{d}\left(\Omega_{i(n+1)}, \Omega_{i(n)}\right) .
\end{aligned}
$$

Taking "lim sup" on both sides of inequality (2) and let $c=\lim _{n \rightarrow \infty} \mathfrak{d}\left(T^{n+1} x, T^{n} x\right)$ by Theorem 1 , it follows that

$$
c \leq c \alpha+(1-\alpha) \limsup _{n \rightarrow \infty} \mathfrak{d}\left(\Omega_{i(n+1)}, \Omega_{i(n)}\right),
$$

and then

$$
c(1-\alpha) \leq(1-\alpha) \limsup _{n \rightarrow \infty} \mathfrak{d}\left(\Omega_{i(n+1)}, \Omega_{i(n)}\right),
$$

dividing $(1-\alpha)$ on both sides of $(3)$, we have

$$
c \leq \limsup _{n \rightarrow \infty} \mathfrak{d}\left(\Omega_{i(n+1)}, \Omega_{i(n)}\right) .
$$

For any $n \in \mathbb{N}$, since $T^{n+1} x \in \Omega_{i(n+1)}$ and $T^{n} x \in A_{i(n)}$, we have $\mathfrak{d}\left(\Omega_{i(n+1)}, \Omega_{i(n)}\right) \leq$ $\mathfrak{d}\left(T^{n+1} x, T^{n} x\right)$ and then

$$
\limsup _{n \rightarrow \infty} \mathfrak{d}\left(\Omega_{i(n+1)}, \Omega_{i(n)}\right) \leq \limsup _{n \rightarrow \infty} \mathfrak{d}\left(T^{n+1} x, T^{n} x\right)(=c) .
$$


By (4) and (5), we have

$$
c=\limsup _{n \rightarrow \infty} \mathfrak{d}\left(\Omega_{i(n+1)}, \Omega_{i(n)}\right) .
$$

Since the cardinal number of $\left\{\Omega_{i}\right\}_{i=1}^{m}$ is " $m$ ", the choice of $\left\{\mathfrak{d}\left(\Omega_{i}, \Omega_{j}\right)\right\}_{i \neq j}$ is at most $\frac{m(m+1)}{2}$ and we have $\limsup _{n \rightarrow \infty} \mathfrak{d}\left(\Omega_{i(n+1)}, \Omega_{i(n)}\right)=\mathfrak{d}\left(\Omega_{\alpha}, \Omega_{\beta}\right)$ for some $1 \leq \alpha \neq \beta \leq m$. Hence

$$
c=\mathfrak{d}\left(\Omega_{\alpha}, \Omega_{\beta}\right) .
$$

Lemma 3. Let $(\mathfrak{X}, \mathfrak{d})$ be a metric space, $\Omega_{i}, i=1,2, \ldots, m$, be nonempty subsets of $\mathfrak{X}$ and $S$ be a subsequence of $\mathbb{N}$ with cardinal number $|S|=\infty$. Consider an alternative map $T: \cup_{i=1}^{m} \Omega_{i} \longrightarrow \cup_{i=1}^{m} \Omega_{i}$ and for any $x \in \cup_{i=1}^{m} \Omega_{i}$, we can find a subsequence $\left\{n_{i}\right\}_{i=0}^{\infty} \subseteq S$ and some subsets $\Omega_{r_{0}}, \Omega_{r_{1}}, \ldots, \Omega_{r_{\eta}} \in\left\{\Omega_{i}\right\}_{i=1}^{m}$ such that

$$
T^{n_{i}} x \in \Omega_{r_{0}} T^{n_{i}+1} x \in \Omega_{r_{1}}, \ldots, T^{n_{i}+\eta} x \in \Omega_{r_{\eta}}, T^{n_{i}+\eta+1} x \in \Omega_{r_{0}}
$$

for some $\eta \in \mathbb{N}$. (Please note that some of $\left\{\Omega_{r_{j}}\right\}_{j=0}^{\eta}$ may be the same.)

Proof. Let $I=\{1,2, \ldots, m\}, x \in \cup_{i=1}^{m} \Omega_{i}$, then $\left\{T^{n} x: n \in S\right\} \subseteq \cup_{i=1}^{m} \Omega_{i}$. By the pigeonhole principle, there exists $S_{0} \subseteq S$ with $\left|S_{0}\right|=\infty$ and $r_{0} \in I$ such that $T^{n_{i}} x \in \Omega_{r_{0}}$ for any $n_{i} \in S_{0}$.

Therefore, we have $T^{n_{i}+1} x \in \cup_{j \neq r_{0}} \Omega_{j}$ for any $n_{i} \in S_{0}$. Since the cardinal number of $S_{0}$ is also infinitely many and the cardinal number of $\left\{\Omega_{j}\right\}_{j \neq r_{0}}$ is only" $m-1$ ", there exists $r_{1} \in I-\left\{r_{0}\right\}$ and $S_{1} \subseteq S_{0}$ with $\left|S_{1}\right|=\infty$ such that $T^{n_{i}+1} x \in \Omega_{r_{1}}$ for any $n_{i} \in S_{1}$.

Similarly, we have $T^{n_{i}+2} x \in \cup_{j \neq r_{1}} \Omega_{j}$ for any $n_{i} \in S_{2}$. Since the cardinal number of $S_{1}$ is also infinitely many and the cardinal number of $\left\{\Omega_{j}\right\}_{j \neq r_{1}}$ is only" $m-1$ ", there exists $r_{2} \in I-\left\{r_{1}\right\}$ and $S_{2} \subseteq S_{1}$ with $\left|S_{2}\right|=\infty$ such that $T^{n_{i}+2} x \in \Omega_{r_{2}}$ for any $n_{i} \in S_{2}$. If $\Omega_{r_{2}}=\Omega_{r_{0}}$, let $\eta=1$, the proof is completed. Else, if $\Omega_{r_{2}} \neq \Omega_{r_{0}}$, will continue the process.

By induction, there exists $r_{3} \in I-\left\{r_{2}\right\}, \ldots, r_{u} \in I-\left\{r_{u-1}\right\}, \ldots, r_{v} \in I-\left\{r_{v-1}\right\}$ and $S_{j+1} \subseteq S_{j}$ with $\left|S_{j+1}\right|=\infty$ for $j=2,3, \ldots, v$ such that $T^{n_{i}+j} x \in \Omega_{r_{j}}$ for any $n_{i} \in S_{j}, j=2,3, \ldots, v-1$. Continuing the process until $\Omega_{r_{v}} \in\left\{\Omega_{r_{j}}\right\}_{j=0}^{v-1}$, then stopping, say $\Omega_{r_{v}}=\Omega_{r_{u}}$ for some $u \in\{0,1,2, \ldots, v-1\}$. In this way, we reset the index of $\Omega_{r_{j}}, j=u, u+1, u+2, \ldots, v$ and let $\eta=v-u$, then

$$
T^{n_{i}} x \in \Omega_{r_{0}}, T^{n_{i}+1} x \in \Omega_{r_{1}}, \ldots, T^{n_{i}+\eta} x \in \Omega_{r_{\eta}} .
$$

Next, we would check that this process would finish by at most $m+1$ steps. Consider the $(m+1)$-th step, since the steps of algorithms is already $m+1$, that is, we produce $m+1$ sets. However, the cardinal number of $\left\{\Omega_{i}\right\}_{i=1}^{m}$ is only $m$, and so, by the pigeonhole principle, there exists two different $r_{j}, r_{k}$ such that $\Omega_{r_{j}}=\Omega_{r_{k}}$. WLOG (Without loss of generalization), we may assume that $j<k$. Reset $k-j-1$ as $\eta$ and reset $\Omega_{r_{j}}$ as $\Omega_{r_{0}}$, reset $\Omega_{r_{j+1}}$ as $\Omega_{r_{1}}$, reset $\Omega_{r_{j+2}}$ as $\Omega_{r_{2}}, \ldots$, reset $\Omega_{r_{k-1}}$ as $\Omega_{r_{k-j-1}}\left(=\Omega_{r_{\eta}}\right)$, reset $\Omega_{k}$ as $\Omega_{\eta+1}=\Omega_{r_{0}}$ such that

$$
T^{n_{i \eta}} x \in \Omega_{r_{0}}, T^{n_{i_{\eta}}+1} x \in \Omega_{r_{1}}, \ldots, T^{n_{i_{\eta}}+\eta} x \in \Omega_{r_{\eta}}, T^{n_{i_{\eta}}+\eta+1} x \in \Omega_{r_{0}} .
$$

The proof is completed. 
Lemma 4. Let $(\mathfrak{X}, \mathfrak{d})$ be a metric space, $\Omega_{i}, i=1,2, \ldots, m$, be nonempty subsets of $\mathfrak{X}$ and $T$ be an alternative contraction. Assume that there exists $x \in \mathfrak{X}$ and a subsequence $\left\{n_{j}\right\} \subseteq \mathbb{N}$ such that $\lim _{j \rightarrow \infty} T^{n_{j}} x$ exists. Let $\lim _{j \rightarrow \infty} T^{n_{j}} x \in A$ for some $A \in\left\{\Omega_{i}\right\}_{i=1}^{m}$ and $T^{n_{j}} x \in \Omega_{n_{j}}$ for some $\Omega_{n_{j}} \in\left\{\Omega_{i}\right\}_{i=1}^{m}$ for any $j \in \mathbb{N}$, then

$$
\limsup _{j \rightarrow \infty} \mathfrak{d}\left(A, \Omega_{n_{j}}\right) \leq \lim _{n \rightarrow \infty} \mathfrak{d}\left(T^{n+1} x, T^{n} x\right)
$$

Proof. Let $p=\lim _{j \rightarrow \infty} T^{n_{j}} x$. Since $\lim _{j \rightarrow \infty} T^{n_{j}} x \in A$ and $T^{n_{j}} x \in \Omega_{n_{j}}$, we have $\mathfrak{d}\left(A, \Omega_{n_{j}}\right) \leq \mathfrak{d}\left(p, T^{n_{j}} x\right)$ for any j. Thus,

$$
\limsup _{j \rightarrow \infty} \mathfrak{d}\left(A, \Omega_{n_{j}}\right) \leq \limsup _{j \rightarrow \infty} \mathfrak{d}\left(p, T^{n_{j}-1} x\right) .
$$

Since $T$ is an alternative contraction, by Theorem $1, \lim _{n \rightarrow \infty} \mathfrak{d}\left(T^{n+1} x, T^{n} x\right)$ exists. It follows that

$$
\begin{aligned}
\limsup _{j \rightarrow \infty} \mathfrak{d}\left(p, T^{n_{j}-1} x\right) & =\limsup _{j \rightarrow \infty} \mathfrak{d}\left(T^{n_{j}} x, T^{n_{j}-1} x\right)\left(\text { since } p=\lim _{j \rightarrow \infty} T^{n_{j}} x\right) \\
& =\lim _{n \rightarrow \infty} \mathfrak{d}\left(T^{n} x, T^{n-1} x\right) .
\end{aligned}
$$

The proof is completed by (6) and (7).

Next, we would use the alternative UC condition as follows:

Definition 11 (Alternative UC condition). Let $(\mathfrak{X}, \mathfrak{d})$ be a metric space and $\Omega_{i}, i=1,2, \ldots, m$ be nonempty subsets of $\mathfrak{X}$. If $\left\{\Omega_{i}\right\}_{i=1}^{m}$ is said to satisfy the UC condition if the following holds. Let $y_{n}^{1}, y_{n}^{v} \in A_{r}$ and $y_{n}^{2}, y_{n}^{3}, \ldots, y_{n}^{v-1} \notin \cup_{j \neq r} \Omega_{j}$ for some positive integer $v \geq 2$. If $\lim _{n \rightarrow \infty} \mathfrak{d}\left(y_{n}^{1}, y_{n}^{2}\right)=\lim _{n \rightarrow \infty} \mathfrak{d}\left(y_{n}^{2}, y_{n}^{3}\right) \ldots=$ $\lim _{n \rightarrow \infty} \mathfrak{d}\left(y_{n}^{v-1}, y_{n}^{v}\right)=\mathfrak{d}(\mathfrak{A}, \mathfrak{B})$ for some $\mathfrak{A}, \mathfrak{B} \in\left\{\Omega_{i}\right\}_{i=1}^{m}$, then $\lim _{n \rightarrow \infty} \mathfrak{d}\left(y_{n}^{1}, y_{n}^{v}\right)=0$.

It is clear that the UC condition of the cyclic map is a special case of the alternative UC Condition.

Lemma 5. Let $(\mathfrak{X}, \mathfrak{d})$ be a metric space, $\Omega_{i}, i=1,2, \ldots, m$ be nonempty subsets of $\mathfrak{X}$ and $T: \cup_{i=1}^{m} \Omega_{i} \longrightarrow$ $\cup_{i=1}^{m} \Omega_{i}$ be an alternative contraction. If there exists $k \in \mathbb{N}$ such that $\lim _{i \rightarrow \infty} T^{n_{i}+j} x=p_{j}$ exists, $j=0,1,2, \ldots, k$ for some $x \in \mathfrak{X}$ and for some subsequence $\left\{n_{i}\right\}_{i=0}^{\infty}$, then the following four conditions are equivalent

(1) $\mathfrak{d}\left(p_{1}, p_{0}\right) \leq \lim _{n \rightarrow \infty} \mathfrak{d}\left(T^{n} x, T^{n+1} x\right)$ ，

(2) $\mathfrak{d}\left(p_{1}, p_{0}\right)=\lim _{n \rightarrow \infty} \mathfrak{d}\left(T^{n} x, T^{n+1} x\right)$,

(3) $\mathfrak{d}\left(p_{k+1}, p_{k}\right) \leq \cdots \leq \mathfrak{d}\left(p_{1}, p_{2}\right) \leq \mathfrak{d}\left(p_{0}, p_{1}\right) \leq \lim _{n \rightarrow \infty} \mathfrak{d}\left(T^{n} x, T^{n+1} x\right)$ ，

(4) $\mathfrak{d}\left(p_{k+1}, p_{k}\right)=\cdots=\mathfrak{d}\left(p_{1}, p_{2}\right)=\mathfrak{d}\left(p_{0}, p_{1}\right)=\lim _{n \rightarrow \infty} \mathfrak{d}\left(T^{n} x, T^{n+1} x\right)$.

Proof. (2) $\Rightarrow(1),(4) \Rightarrow(3),(4) \Rightarrow(2)$ and $(3) \Rightarrow(1)$ are obvious. The remainder part is to prove $(1) \Rightarrow(4)$. By Corollary 2.4, $\left\{\mathfrak{d}\left(T^{n+1} x, T^{n} x\right)\right\}_{n \in \mathbb{N}}$ is a decreasing sequence, we have

$$
\lim _{n \rightarrow \infty} \mathfrak{d}\left(T^{n+1} x, T^{n} x\right) \leq \mathfrak{d}\left(T^{n_{i}+k} x, T^{n_{i}+k-1} x\right) \leq \cdots \leq \mathfrak{d}\left(T^{n_{i}+2} x, T^{n_{i}+1} x\right) \leq \mathfrak{d}\left(T^{n_{i}+1} x, T^{n_{i}} x\right) .
$$

Since $p_{j}=\lim _{i \rightarrow \infty} T^{n_{i}+j} x$ exist, $j=1,2, \ldots, k$ and then taking lim on inequality (9). It follows that

$$
\lim _{n \rightarrow \infty} \mathfrak{d}\left(T^{n+1} x, T^{n} x\right) \leq \mathfrak{d}\left(p_{k}, p_{k-1}\right) \leq \cdots \leq \mathfrak{d}\left(p_{2}, p_{1}\right) \leq \mathfrak{d}\left(p_{1}, p_{0}\right) .
$$

By assumption (1),

$$
\mathfrak{d}\left(p_{1}, p_{0}\right) \leq \lim _{n \rightarrow \infty} \mathfrak{d}\left(T^{n+1} x, T^{n} x\right)
$$


Combining (9) and (10), we have

$$
\mathfrak{d}\left(p_{k+1}, p_{k}\right)=\cdots=\mathfrak{d}\left(p_{1}, p_{2}\right)=\mathfrak{d}\left(p_{0}, p_{1}\right)=\lim _{n \rightarrow \infty} \mathfrak{d}\left(T^{n} x, T^{n+1} x\right) .
$$

Theorem 3. Let $(\mathfrak{X}, \mathfrak{d})$ be a metric space with $U C$ condition, $\Omega_{i}, i=1,2, \ldots, m$ be nonempty subsets of $\mathfrak{X}$ and $T: \cup_{i=1}^{m} \Omega_{i} \longrightarrow \cup_{i=1}^{m} \Omega_{i}$ be an ADC with the UC condition. If there exists $x \in X$ and a subsequence $\left\{n_{i}\right\}_{i=0}^{\infty}$ of $\mathbb{N}$ such that $p_{j}=\lim _{i \rightarrow \infty} T^{n_{i}+j} x, j=0,1,2, \ldots, m$ with $\mathfrak{d}\left(p_{1}, p\right) \leq \lim _{i \rightarrow \infty} \mathfrak{d}\left(T^{n} x, T^{n+1} x\right)$, then there exist different $k, \ell \in \mathbb{N}$ such that $p_{k}=p_{\ell}$.

Proof. Since $T: \cup_{i=1}^{m} \Omega_{i} \longrightarrow \cup_{i=1}^{m} \Omega_{i}$ is an ADC and there exists a subsequence $\left\{n_{i}\right\}_{i=0}^{\infty}$ such that $p_{j}=\lim _{i \rightarrow \infty} T^{n_{i}+j} x, j=0,1,2, \ldots, m$ with $\mathfrak{d}\left(p_{1}, p\right) \leq \lim _{i \rightarrow \infty} \mathfrak{d}\left(T^{n} x, T^{n+1} x\right)$, by Lemma 5 , we have

$$
\mathfrak{d}\left(p_{k+1}, p_{k}\right)=\cdots=\mathfrak{d}\left(p_{1}, p_{2}\right)=\mathfrak{d}\left(p_{0}, p_{1}\right)=\lim _{n \rightarrow \infty} \mathfrak{d}\left(T^{n} x, T^{n+1} x\right) .
$$

Since $p_{0}, p_{1}, \ldots, p_{m} \in \cup_{i=1}^{m} \Omega_{i}$, the cardinal number of $\left\{p_{i}\right\}_{i=0}^{m}$ is $m+1$ and the cardinal number of $\left\{\Omega_{i}\right\}_{i=1}^{m}$ is $m$, by the pigeonhole principle, there exist two different $k, \ell \in \mathbb{N}$ such that $p_{k}, p_{\ell} \in \Omega_{j}$ for some $j$. WLOG, we assume that $k<\ell$, then

$$
\mathfrak{d}\left(p_{\ell}, p_{\ell-1}\right)=\cdots=\mathfrak{d}\left(p_{k+2}, p_{k+1}\right)=\mathfrak{d}\left(p_{k+1}, p_{k}\right)=\lim _{n \rightarrow \infty} \mathfrak{d}\left(T^{n} x, T^{n+1} x\right) .
$$

By Theorem 2, we have $\lim _{n \rightarrow \infty} \mathfrak{d}\left(T^{n} x, T^{n+1} x\right)=\mathfrak{d}(\mathfrak{A}, \mathfrak{B})$ for some $\mathfrak{A}, \mathfrak{B} \in\left\{\Omega_{i}\right\}_{i=1}^{m}$ and it then $p_{k}=p_{\ell}$ by (11) and the UC condition.

Theorem 4. Let $(\mathfrak{X}, \mathfrak{d})$ be a metric space, $\Omega_{i}, i=1,2, \ldots, m$ be nonempty subsets of $\mathfrak{X}$. Suppose $T$ : $\cup_{i=1}^{m} \Omega_{i} \longrightarrow \cup_{i=1}^{m} \Omega_{i}$ is a ALC, if for any $x \in \cup_{i=1}^{m} \Omega_{i}$, there exists a subsequence $\left\{n_{i}\right\}_{i=1}^{\infty}$ of $\mathbb{N}$ with $p_{j}=$ $\lim _{i \rightarrow \infty} T^{n_{i}+j}$ x existing for $j=0,1,2, \ldots, m$, then $p_{j}=T^{j} p_{0}, j=1,2, \ldots, k$.

Proof. For any $j=1,2, \ldots, k$,

$$
\begin{aligned}
p_{j} & =\lim _{i \rightarrow \infty} T^{n_{i}+j} x \\
& \left.=\lim _{i \rightarrow \infty} T\left(T^{n_{i}+j-1} x\right)\right) \\
& \left.=T\left(\lim _{i \rightarrow \infty} T^{n_{i}+j-1} x\right)\right) \text { (since } T \text { is a ALC) } \\
& =T p_{j-1} .
\end{aligned}
$$

By induction, we have $p_{j}=T^{j} p_{0}$.

Theorem 5. Let $(\mathfrak{X}, \mathfrak{d})$ be a metric space, $\Omega_{i}, i=1,2, \ldots, m$ be nonempty subsets of $\mathfrak{X}$. Suppose $T$ : $\cup_{i=1}^{m} \Omega_{i} \longrightarrow \cup_{i=1}^{m} \Omega_{i}$ is an alternative contraction, if there exists $x \in \cup_{i=0}^{m} \Omega_{i}$ and a subsequence $\left\{n_{i}\right\}_{i=1}^{\infty}$ of $\mathbb{N}$ such that $p_{j}=\lim _{i \rightarrow \infty} T^{n_{i}+j} x$ exists, $j=0,1,2, \ldots, m$, then there exist some best proximity points of $T$.

Proof. Since $T$ is an AC and by Theorem 4, we have

$$
p_{j}=T^{j} p_{0}, j=1,2, \ldots, m .
$$

Moreover, by the definitions of ALC,

$$
\mathfrak{d}(T p, p) \leq \lim _{n \rightarrow \infty} \mathfrak{d}\left(T^{n+1} x, T^{n} x\right)
$$


We can derive by (12) and it leads

$$
\mathfrak{d}\left(p_{1}, p\right) \leq \lim _{n \rightarrow \infty} \mathfrak{d}\left(T^{n+1} x, T^{n} x\right) .
$$

By Theorem 3, we have

$$
p_{\kappa}=p_{\rho} \text { for some } \kappa<\rho .
$$

Moreover, by Lemma 5, we observe

$$
\mathfrak{d}\left(p_{\kappa}, p_{\kappa+1}\right)=\mathfrak{d}\left(p_{\kappa+1}, p_{\kappa+2}\right)=\ldots=\mathfrak{d}\left(p_{\rho-1}, p_{\rho}\right)=\lim _{n \rightarrow \infty} \mathfrak{d}\left(T^{n+1} x, T^{n} x\right) .
$$

Let $p=p_{\kappa}, \ell=\rho-\kappa$ and $q_{j}=p_{\kappa+j}, j=1,2, \ldots, \ell$, it then

$$
q_{j}=p_{\kappa+j}=T^{j} p_{\kappa}=T^{j} p
$$

by (12). By (13) above, we can derive that

$$
p=p_{\kappa}=p_{\rho}=T^{\ell} p .
$$

One has $\lim _{n \rightarrow \infty} \mathfrak{d}\left(T^{n+1} x, T^{n} x\right)=\mathfrak{d}\left(A_{\alpha}, A_{\beta}\right)$ by Lemma 5, this implies

$$
\mathfrak{d}\left(p, q_{1}\right)=\mathfrak{d}\left(q_{1}, q_{2}\right)=\ldots=\mathfrak{d}\left(q_{\ell-1}, q_{\ell}\right)=\mathfrak{d}\left(A_{\alpha}, A_{\beta}\right) .
$$

Then by (14) and (16),

$$
\mathfrak{d}(p, T p)=\mathfrak{d}\left(T p, T^{2} p\right)=\ldots=\mathfrak{d}\left(T^{\ell-1} p, T^{\ell} p\right)=\mathfrak{d}\left(A_{\alpha}, A_{\beta}\right) .
$$

Let $T^{j} p \in \Omega_{r_{j}}$ for some $\Omega_{r_{j}} \in\left\{\Omega_{i}\right\}_{i=1}^{m}, j=0,1, \ldots, \ell$. Hence

$$
\begin{aligned}
& \mathfrak{d}\left(T^{j+2} p, T^{j+1} p\right) \leq \tau \mathfrak{d}\left(T^{j+1} p, T^{j} p\right)+(1-\tau) \mathfrak{d}\left(\Omega_{r_{j+1}}, \Omega_{r_{j}}\right) \\
\Longrightarrow & \mathfrak{d}\left(\Omega_{\alpha}, \Omega_{\beta}\right) \leq \tau \mathfrak{d}\left(\Omega_{\alpha}, \Omega_{\beta}\right)+(1-\tau) \mathfrak{d}\left(\Omega_{r_{j+1}}, \Omega_{r_{j}}\right)(\text { by }(17)) \\
\Longrightarrow & (1-\tau) \mathfrak{d}\left(\Omega_{\alpha}, \Omega_{\beta}\right) \leq(1-\tau) \mathfrak{d}\left(\Omega_{r_{j+1}}, A_{r_{j}}\right) \\
\Longrightarrow & \mathfrak{d}\left(\Omega_{\alpha}, \Omega_{\beta}\right) \leq \mathfrak{d}\left(\Omega_{r_{j+1}}, \Omega_{r_{j}}\right) .
\end{aligned}
$$

Moreover, since $T^{j+1} p \in \Omega_{r_{j+1}}$ and $T^{j} p \in \Omega_{r_{j}}$, we have

$$
\mathfrak{d}\left(\Omega_{r_{j+1}}, \Omega_{r_{j}}\right) \leq \mathfrak{d}\left(T^{j+1} p_{0}, T^{j} p_{0}\right)=\mathfrak{d}\left(\Omega_{\alpha}, \Omega_{\beta}\right) .
$$

Combining (18) and (19), it follows that

$$
\mathfrak{d}\left(\Omega_{r_{j+1}}, \Omega_{r_{j}}\right)=\mathfrak{d}\left(\Omega_{\alpha}, \Omega_{\beta}\right)
$$

for $j=0,1,2, \ldots, \ell$. By (15), (17) and (20), we have $p$ is a best proximity point of $T$.

Theorem 6. Let $(\mathfrak{X}, \mathfrak{d})$ be a compact metric space and $\Omega_{i}, i=1,2, \ldots, m$ be nonempty closed subsets of $(\mathfrak{X}, \mathfrak{d})$. Suppose $T: \cup_{i=1}^{m} \Omega_{i} \longrightarrow \cup_{i=1}^{m} \Omega_{i}$ is an alternative map, then there exists a best proximity point of $T$.

Proof. By Lemma 3, for any integer $k$, there exists a subsequence $\left\{n_{i}\right\}_{i=0}^{\infty} \subseteq \mathbb{N}$ such that

$$
T^{n_{i}} x \in \Omega_{r_{0}} T^{n_{i}+1} x \in \Omega_{r_{1}}, \ldots, T^{n_{i}+k} x \in \Omega_{r_{k}}
$$


for all $i$. For any $j=0,1,2, \ldots, k$, since $\mathfrak{X}$ is compact, we have $\lim _{i \rightarrow \infty} T^{n_{i}+j} x$ exists. Moreover, since $\Omega_{r_{j}} \in\left\{\Omega_{i}\right\}_{i=1}^{m}$ and $\Omega_{i}$ is closed, $i=1,2, \ldots, m$, it follows that $\lim _{i \rightarrow \infty} T^{n_{i}+j} x$ exists and

$$
\lim _{i \rightarrow \infty} T^{n_{i}+j} x \in \Omega_{r_{j}}, j=0,1,2, \ldots, k .
$$

By Theorem 5, there exists a best proximity point of $T$.

Corollary 2. Let $(\mathfrak{X}, \mathfrak{d})$ be a compact (or weakly countable compact) metric space, $\Omega_{i}, i=1,2, \ldots, m$ be nonempty closed subsets of $(\mathfrak{X}, \mathfrak{d})$. Suppose $T: \cup_{i=1}^{m} \Omega_{i} \longrightarrow \cup_{i=1}^{m} \Omega_{i}$ is an $A C$, then there exists a best proximity point of $T$.

Proof. This corollary can be derived by Theorem 5 and Theorem 6 immediately.

Theorem 7. Let $(\mathfrak{X}, \mathfrak{d})$ be a complete metric space, $\Omega_{i}, i=1,2, \ldots$, m be nonempty closed subsets of $(\mathfrak{X}, \mathfrak{d})$. Suppose $T: \cup_{i=1}^{m} \Omega_{i} \longrightarrow \cup_{i=1}^{m} \Omega_{i}$ is an AC with UC condition, then there exists a best proximity point of $T$.

Proof. Since $T^{n_{i}+j} x \in \cup_{i=1}^{m} \Omega_{i}, j=1,2, \ldots, m+1$, by the pigeonhole principle, there exist $1 \leq \mu<v \leq$ $m+1$ such that $T^{n_{i}+\mu} x, T^{n_{i}+v} x \in \Omega_{k}$ for any $i \in \mathbb{N}$ and some $k \in\{1,2, \ldots, m\}$. Moreover, since $T$ is an alternative contraction, by Theorem 1 , we have

$$
\begin{aligned}
\lim _{i \rightarrow \infty} \mathfrak{d}\left(T^{n_{i}+v} x, T^{n_{i}+v-1} x\right) & =\lim _{i \rightarrow \infty} \mathfrak{d}\left(T^{n_{i}+v-1} x, T^{n_{i}+v-2} x\right) \\
& =\lim _{i \rightarrow \infty} \mathfrak{d}\left(T^{n_{i}+v-2} x, T^{n_{i}+v-3} x\right) \\
& =\cdots \\
& =\lim _{i \rightarrow \infty} \mathfrak{d}\left(T^{n_{i}+\mu+1} x, T^{n_{i}+\mu} x\right)=\lim _{n \rightarrow \infty} \mathfrak{d}\left(T^{n+1} x, T^{n} x\right) .
\end{aligned}
$$

By UC condition, $\lim _{n \rightarrow \infty} \mathfrak{d}\left(T^{n_{i+v}} x, T^{n_{i+\mu}} x\right)=0$. This can imply lead to a Cauchy sequence. That is

$$
\lim _{i \rightarrow \infty} T^{n_{i}} x=z
$$

for some $z \in X$. By Theorem 5 , there exists a best proximity point of $T$.

Acknowledgments: The authors would like to thank the associate editor for his/her comments that helped us improve this article.

Conflicts of Interest: The author declares no conflict of interest.

\section{References}

1. Kirk, W.A.; Srinavasan, P.S.; Veeramani, P. Fixed Points for mapping satisfying cyclical contractive conditions. Fixed Point Theory 2003, 4, 79-89.

2. Eldred, A.A.; Veeramani, P. Existence and Convergence of Best Proximity Points. J. Math. Anal. Appl. 2006, 323, 1001-1006. [CrossRef]

3. Suzuki, T.; Kikkawa, M.; Vetro, C. The Existence of Best Proximity Points in Metric Spaces with the Property UC. Nonlinear Anal. Theory Methods Appl. 2009, 71, 2918-2926. [CrossRef]

4. Fan, K. Extensions of Two Fixed Point Theorems of F. E. Browder. Mathematische Zeitschrift 1969, 112, $234-240$. [CrossRef]

5. Ravindranadh Babu, G.V.; Dula Tolera, M. Fixed Points of Generalized $(\alpha, \psi, \varphi)$-Rational Contractive Mappings in $\alpha$-Complete Metric Spaces. Fasciculi Mathematici, Sciendo, 23 October 2018. Available online: content.sciendo.com/view/journals/fascmath/59/1/article-p13.xml (accessed on 13 April 2019).

6. Bajpai, K.; Gandhi, M.; Anjum, S. Fixed Point Result Satisfying New Contractive Condition in Complete G-Metric Space. In AIP Conference Proceedings; AIP Publishing: Melville, NY, USA, 2019; p. 020046. [CrossRef] 
7. Di Bari, C.; Suzuki, T.; Vetro, C. Best Proximity Points for Cyclic Meir-Keeler Contractions. Nonlinear Anal. Theory Methods Appl. 2008, 69, 3790-3794. [CrossRef]

8. Chen, C.-M. Fixed Point Theory of Cyclical Generalized Contractive Conditions in Partial Metric Spaces. Fixed Point Theory Appl. 2013, 2013, 17. [CrossRef]

9. Du, W.-S. On Caristi-Type Mappings without Lower Semicontinuity Assumptions. J. Fixed Point Theory Appl. 2015, 17, 733-752. [CrossRef]

10. Mirdamadi, F.; Soltani, Z. Some Results on Best Proximity Points of Cyclic Meir-Keeler Contraction Mappings. Filomat 2018, 32, 2081-2089. [CrossRef]

11. Kaliaj, S.B. A Kannan-Type Fixed Point Theorem for Multivalued Mappings with Application. J. Anal. 2018. [CrossRef]

12. Klanarong, C.; Chaobankoh, T. Best Proximity Point Theorems for a Berinde MT-Cyclic Contraction on a Semisharp Proximal Pair. Int. J. Math. Math. Sci. 2018, 2018, 1-7. [CrossRef]

13. Kongban, C.; Kumam, P. Some Random Coupled Best Proximity Points for a Generalized $\omega$-Cyclic Contraction in Polish Spaces. Fasciculi Mathematici 2017, 59, 91-105. [CrossRef]

14. Kumari, P.S.; Nantadilok, J.; Sarwar, M. Fixed Point Theorems for a Class of Generalized Weak Cyclic Compatible Contractions. Fixed Point Theory Appl. 2018, 2018, 13. [CrossRef]

15. Lin, I.-J., Chang, Y.-L. Some new generalizations of Karapinar's theorems. Int. J. Math. Anal. 2014, 8, 957-966. [CrossRef]

16. Mongkolkeha, C.; Poom, K. Best Proximity Point Theorems for Cyclic Contractions Mappings. In Background and Recent Developments of Metric Fixed Point Theory; Chapman and Hall/CRC: Boca Raton, FL, USA, 2017; pp. 201-228. [CrossRef]

17. Popa, V. A General Fixed Point Theorem For Implicit Cyclic Multi-Valued Contraction Mappings. Ann. Math. Silesianae 2015, 29, 119-129. [CrossRef]

18. Sanhan, S.; Chirasak, M. Convergence and Best Proximity Points for Berinde's Cyclic Contraction with Proximally Complete Property. Math. Methods Appl. Sci. 2016, 39, 4866-4873. [CrossRef]

19. Abkar, A.; Moezzifar, N.; Azizi, A.; Shahzad, N. Best Proximity Point Theorems for Cyclic Generalized Proximal Contractions. Fixed Point Theory Appl. 2016, 2016, 66. [CrossRef]

20. Ardsalee, P.; Saejung, S. Best Proximity Point Theorems via Fixed Point Theorems for Multivalued Mappings. Fixed Point Theory Appl. 2016, 2016, 29. [CrossRef]

21. Ayari, M.I. Best Proximity Point Theorems for Generalized $\alpha$ - $\beta$-Proximal Quasi-Contractive Mappings. Fixed Point Theory Appl. 2017, 2017, 16. [CrossRef]

22. Du, W.-S. On Coincidence Point and Fixed Point Theorems for Nonlinear Multivalued Maps. Topol. Its Appl. 2012, 159, 49-56. [CrossRef]

23. Felhi, A. Best Proximity Points in Partially Ordered Metric Spaces. Math. Methods Appl. Sci. 2018, 41, 140-143. [CrossRef]

24. Felhi, A. A Note on 'Convergence and Best Proximity Points for Berinde's Cyclic Contraction with Proximally Complete Property. Math. Methods Appl. Sci. 2017, 41, 140-143. [CrossRef]

25. Haddadi, M.R. Existence and Convergence Theorems for Best Proximity Points. Asian-Eur. J. Math. 2017, 11, 1850005. [CrossRef]

26. Kumar, A.; Rathee, S.; Dhingra, K. Existence of Best Proximity Points for Generalized $(\alpha-\eta)$ - Rational Proximal Contraction. Int. J. Math. Trends Technol. 2017, 52, 528-536. [CrossRef]

27. Liu, H.; Fan, X.; Yan, L.; Wang, Z. On the Existence of Coupled Best Proximity Point and Best Proximity Point for Suzuki Type Alpha+-Theta-Proximal Multivalued Mappings. J. Nonlinear Sci. Appl. 2017, 10, 1801-1819. [CrossRef]

28. Mongkolkeha, C.; Cho, Y.J.; Kumam, P. Best Proximity Points for Generalized Proximal C-Contraction Mappings in Metric Spaces with Partial Orders. J. Inequalities Appl. 2013, 2013, 94. [CrossRef]

29. Pitea, A. Best Proximity Results on Dualistic Partial Metric Spaces. Symmetry 2019, 11, 306. [CrossRef]

30. Shukri, S.A.; Khan, A.R. Best Proximity Points in Partially Ordered Metric Spaces. Adv. Fixed Point Theory 2018. [CrossRef]

31. Sartaj, A.; Mujahid, A.; Safeer, H.K. Best Proximity Point Theorems for $F_{\rho}$-Proximal Contraction in Modular Function Spaces. Adv. Fixed Point Theory 2018. [CrossRef]

32. Shatanawi, W.; Ariana, P. Best Proximity Point and Best Proximity Coupled Point in a Complete Metric Space with (P)-Property. Filomat 2015, 29, 63-74. [CrossRef] 
33. Suparatulatorn, R.; Cholamjiak, W.; Suantai, S. Existence and Convergence Theorems for Global Minimization of Best Proximity Points in Hilbert Spaces. Acta Appl. Math. 2019. [CrossRef]

34. Veeramani, P.; Rajesh, S. Best Proximity Points. Nonlinear Anal. 2014, 1-32. [CrossRef]

(C) 2019 by the author. Licensee MDPI, Basel, Switzerland. This article is an open access article distributed under the terms and conditions of the Creative Commons Attribution (CC BY) license (http:/ / creativecommons.org/licenses/by/4.0/). 